\title{
SOME WEIGHTED NORM INEQUALITIES FOR THE FOURIER TRANSFORM OF FUNCTIONS WITH VANISHING MOMENTS
}

\author{
CORA SADOSKY AND RICHARD L. WHEEDEN
}

\begin{abstract}
Weighted $L^{p}$ norm inequalities are derived between a function and its Fourier transform in case the function has vanishing moments up to some order. For weights of the form $|x|^{\gamma}$, the results concern values of $\gamma$ which are outside the range which is normally considered.
\end{abstract}

1. Introduction. Weighted norm inequalities for the Fourier transform with power weights have natural constraints on the exponents, as indicated in Pitt's theorem [6], which asserts for example that

$$
\int_{-\infty}^{\infty}|\hat{f}(x)|^{p}|x|^{-\gamma+p-2} d x \leqslant C \int_{-\infty}^{\infty}|f(x)|^{p}|x|^{\gamma} d x
$$

if $1<p<\infty$ and $\max \{0, p-2\} \leqslant \gamma<p-1$. The result fails for $\gamma$ outside this range. We will show, however, that (1) holds for $\gamma>p-1, \gamma \neq k p-1$ for $k=1,2, \ldots$, provided that enough moments of $f$ vanish. For example, an immediate consequence of Theorem 1 below is that (1) is valid for $p-1<\gamma<2 p-1$ for all $f$ having mean value zero (cf. [2], where analytic functions in the unit circle are considered). The case $\gamma=p-1$ is excluded, even with this restriction on $f$, as shown by the counterexample in $\S 5$.

We work with functions in $\mathscr{S}_{0,0}$, the class of Schwartz functions whose Fourier transforms have compact support not containing the origin. Note that all the moments of a function in $\mathscr{S}_{0,0}$ vanish:

$$
\int_{-\infty}^{\infty} f(x) x^{j} d x=0, \quad j=0,1,2, \ldots, f \in \mathscr{S}_{0,0} .
$$

$\mathscr{S}_{0,0}$ is dense in all the weighted spaces that we will consider, and the Fourier transform operator has a natural extension to functions (not necessarily locally integrable) in these spaces: see $\$ 4$.

In what follows, if $1<p<\infty, A_{p}$ stands for the class of nonnegative, locally integrable functions $w$ on $\mathbf{R}^{1}$ such that

$$
\left(\frac{1}{|I|} \int_{I} w(x) d x\right)\left(\frac{1}{|I|} \int_{I} w(x)^{-1 /(p-1)} d x\right)^{p-1} \leqslant A<\infty
$$

for all intervals $I \subset \mathbf{R}^{1}$.

Received by the editors December 5, 1985.

1980 Mathematics Subject Classification (1985 Revision). Primary 42A68, 44A50.

The first author was supported in part by NSF grant \# RII-83-10298 and the second author by NSF grant \#DMS-85-03316. 
THEOREM 1. If $1<p<\infty$ and $w \in A_{p}$, then

$$
\int_{-\infty}^{\infty}|\hat{f}(x)|^{p} \frac{1}{|x|} w\left(\frac{1}{x}\right) \frac{d x}{|x|} \leqslant C \int_{-\infty}^{\infty}|f(x)|^{p}|x|^{p} w(x) d x
$$

for all $f$ with $\int_{-\infty}^{\infty} f d x=0$, where $C$ is independent of $f$.

Inequality (1) for $p-1<\gamma<2 p-1$ follows from (2) since $|x|^{\gamma-p} \in A_{p}$ if $-1<\gamma-p<p-1$. Counterexamples for $\gamma=p-1$, etc., are discussed in $\S 5$.

More generally, we have

TheOREM 1a. If $1<p \leqslant q<\infty, w^{q / p} \in A_{1+q / p^{\prime}}, 1 / p+1 / p^{\prime}=1$, and $k$ is a positive integer, then

$$
\text { (3) }\left(\int_{-\infty}^{\infty}\left|\frac{\hat{f}(x)}{x^{k-1}}\right|^{q}\left(\frac{1}{|x|} w\left(\frac{1}{x}\right)\right)^{q / p} \frac{d x}{|x|}\right)^{1 / q} \leqslant C\left(\int_{-\infty}^{\infty}|f(x)|^{p}|x|^{k p} w(x) d x\right)^{1 / p}
$$

for all $f \in \mathscr{S}_{0,0}$, with $C$ independent of $f$.

The condition on $w$ above is easily seen to be equivalent to

$$
\left(\frac{1}{|I|} \int_{I} w^{q / p} d x\right)^{p / q}\left(\frac{1}{|I|} \int_{I} w^{-1 /(p-1)} d x\right)^{p-1} \leqslant A<\infty .
$$

Moreover, it is equivalent to assuming that $w \in A_{p} \cap \mathrm{RH}_{q / p}$, where $w \in \mathrm{RH}_{r}$, $r>1$, means that $w$ satisfies the reverse Hölder condition

$$
\left(\frac{1}{|I|} \int_{I} w^{r} d x\right)^{1 / r} \leqslant C\left(\frac{1}{|I|} \int_{I} w d x\right)
$$

with $C$ independent of $I$.

The proof of these results is extremely simple and based only on Hardy's inequalities and some properties of $A_{p}$ weights. As a consequence, the results hold under considerably weaker hypotheses than $w^{q / p} \in A_{1+q / p^{\prime}}$. For example, Theorem 1a together with the density of $\mathscr{S}_{0,0}$ in $L^{p}\left(|x|^{k p} w\right)$ are valid if $w$ is locally integrable and both of the following hold:

$$
\begin{aligned}
& \left(\int_{|x|>s} \frac{w(x)^{q / p}}{|x|^{1+q / p^{\prime}}} d x\right)^{p / q}\left(\int_{|x|<s} w(x)^{-1 /(p-1)} d x\right)^{p-1} \leqslant C \\
& \left(\int_{|x|<s}|x|^{q / p-1} w(x)^{q / p} d x\right)^{p / q}\left(\int_{|x|>s} \frac{w(x)^{-1 /(p-1)}}{|x|^{p^{\prime}}} d x\right)^{p-1} \leqslant C
\end{aligned}
$$

for all $s>0$. This will follow for Theorem 1a by combining the comments in Remarks 1a, $2 \mathrm{a}$ of $\S 2$. For the density, it follows from [5]; see the end of $\S 4$.

Theorem 1a also has translated versions. To obtain these, apply Theorem la to the function $e^{i x b} f(x+a)$ and the weight $w(x+a)$, noting that the condition $w^{q / p} \in$ $A_{1+q / p^{\prime}}$ is translation invariant. Then translating the integral which arises on the 
right of (3) by $a$ and the integral which arises on the left by $b$, we obtain

$$
\begin{gathered}
\left(\int_{-\infty}^{\infty}\left|\frac{\hat{f}(x)}{x-b}\right|^{q}\left[\frac{1}{x-b} w\left(a+\frac{1}{|x-b|}\right)\right]^{q / p} \frac{d x}{|x-b|}\right)^{1 / q} \\
\leqslant C\left(\int_{-\infty}^{\infty}|f(x)|^{p}|x-a|^{k p} w(x) d x\right)^{1 / p}
\end{gathered}
$$

with $C$ independent of $f, a$, and $b$, provided that $w^{q / p} \in A_{1+q / p^{\prime}}$ and all the moments of $e^{i x b} f(x)$ vanish. Instead of assuming that $w^{q / p} \in A_{1+q / p^{\prime}}$, we could assume only that (5) holds for $w(x+a)$.

In $n$ dimensions, we have the following result:

TheOREM 2. Let $n \geqslant 1,1<p \leqslant q<\infty, \beta=(n-1) p-n$, and $w$ be a function on $\mathbf{R}^{1}$ such that the function $\tilde{w}(\rho)=|\rho|^{-(\beta+1)} w(\rho)$ satisfies $\tilde{w}^{q / p} \in A_{1+q / p^{\prime}}\left(\mathbf{R}^{1}\right)$. If $k$ is a positive integer and $f \in \mathscr{S}_{0,0}$, then

$$
\left(\int_{\mathbf{R}^{n}}\left(\frac{|\hat{f}(x)|}{|x|^{k-1}}\right)^{q}\left[|x|^{\beta} w\left(\frac{1}{|x|}\right)\right]^{q / p} \frac{d x}{|x|^{n}}\right)^{1 / q} \leqslant C\left(\int_{\mathbf{R}^{n}}|f(x)|^{p}|x|^{k p} w(|x|) d x\right)^{1 / p}
$$

with $C$ independent of $f$.

Again, the conclusion of Theorem 2 holds under a weaker assumption on $\tilde{w}$; it is enough to assume that $\tilde{w}$ satisfies the analogue of (5) with all integrations restricted to positive values of the variable of integration, i.e., to assume

$$
\begin{aligned}
& \left(\int_{s}^{\infty} \frac{\tilde{w}(\rho)^{q / p}}{\rho^{1+q / p^{\prime}}} d \rho\right)^{p / q}\left(\int_{0}^{s} \tilde{w}(\rho)^{-1 /(p-1)} d \rho\right)^{p-1} \leqslant C \\
& \left(\int_{0}^{s} \rho^{q / p-1} \tilde{w}(\rho)^{q / p} d \rho\right)^{p / q}\left(\int_{s}^{\infty} \frac{\tilde{w}(\rho)^{-1 /(p-1)}}{\rho^{p^{\prime}}} d \rho\right)^{p-1} \leqslant C
\end{aligned}
$$

for all $s>0$.

In $\S 2$, we list some auxiliary weighted inequalities used in the proofs of Theorems 1 and $1 \mathrm{a}$; the proofs themselves are given in $\S 3$. In $\$ 4$, we discuss the extensions by continuity of $\hat{f}$ for general $f \in L^{p}\left(|x-a|^{k p} w\right)$, and in $\S 5$ we consider a counterexample for power weights. Theorem 2 is proved in $\S 6$.

Throughout the paper, $C$ stands for a constant which may be different at different occurrences, and $p^{\prime}$ denotes the conjugate index of $p: 1 / p+1 / p^{\prime}=1,1<p<\infty$.

2. Basic inequalities. As mentioned in the introduction, the proofs are based entirely on Hardy's inequality and a few properties of $A_{p}$ weights. In the next two lemmas, we summarize the facts we shall use.

Lemma A. If $w \in A_{p}\left(\mathbf{R}^{1}\right), 1<p<\infty$, and $\alpha \geqslant p$, then

$$
\int_{|t|>s} t^{-\alpha} w(t) d t \leqslant C s^{-\alpha} \int_{|t|<s} w(t) d t, \quad s>0,
$$

with $C$ independent of $s$.

For a proof, see (2.3) in [3]. 
LEMMA B (HARDY'S INEQUALITIES). If $1<p \leqslant q<\infty$ and $u$ and $v$ are nonnegative on $(0, \infty)$, then the inequality

$$
\left(\int_{0}^{\infty}\left(\int_{x}^{\infty} f(t) d t\right)^{q} u(x) d x\right)^{1 / q} \leqslant C\left(\int_{0}^{\infty} f(x)^{p} v(x) d x\right)^{1 / p}
$$

holds for every $f \geqslant 0$ if and only if

$$
\sup _{r>0}\left(\int_{0}^{r} u(x) d x\right)^{1 / q}\left(\int_{r}^{\infty} v(x)^{-p^{\prime} / p} d x\right)^{1 / p^{\prime}}<\infty .
$$

Similarly, the inequality

$$
\left(\int_{0}^{\infty}\left(\int_{0}^{x} f(t) d t\right)^{q} u(x) d x\right)^{1 / q} \leqslant C\left(\int_{0}^{\infty} f(x)^{p} v(x) d x\right)^{1 / p}
$$

for every $f \geqslant 0$ is equivalent to

$$
\sup _{r>0}\left(\int_{r}^{\infty} u(x) d x\right)^{1 / q}\left(\int_{0}^{r} v(x)^{-p^{\prime} / p} d x\right)^{1 / p^{\prime}}<\infty .
$$

For a proof, see [1].

We will use Lemmas A and B to prove the next four lemmas, which are the specific inequalities needed for the theorems in the introduction. Since the case $q=p$ is somewhat simpler, we will consider it separately.

Lemma 1. If $1<p<\infty$ and $w \in A_{p}$, then

$$
\int_{-\infty}^{\infty}\left(\int_{|t|>|x|} g(t) d t\right)^{p}|x|^{p-2} w\left(\frac{1}{x}\right) d x \leqslant C \int_{-\infty}^{\infty} g(x)^{p}|x|^{2 p-2} w\left(\frac{1}{x}\right) d x
$$

for $g \geqslant 0$.

Proof. The expression on the left side of (14) is at most $2^{p}$ times

$$
\int_{0}^{\infty}\left(\int_{x}^{\infty}\right)^{p}+\int_{0}^{\infty}\left(\int_{-\infty}^{-x}\right)^{p}+\int_{-\infty}^{0}\left(\int_{-x}^{\infty}\right)^{p}+\int_{-\infty}^{0}\left(\int_{-\infty}^{x}\right)^{p}=\mathrm{I}+\mathrm{II}+\mathrm{III}+\mathrm{IV} .
$$

We want to show that each of these is bounded by the term on the right side of (14).

We have

$$
\mathrm{I}+\mathrm{III}=\int_{0}^{\infty}\left(\int_{x}^{\infty} g(t) d t\right)^{p} x^{p-2}\left[w\left(\frac{1}{x}\right)+w\left(-\frac{1}{x}\right)\right] d x .
$$

By Hardy's inequality (10) with $q=p$, this will be bounded by

$$
C \int_{0}^{\infty} g(x)^{p} x^{2 p-2} w\left(\frac{1}{x}\right) d x
$$

provided that (11) is satisfied for

$$
u(x)=x^{p-2}\left[w\left(\frac{1}{x}\right)+w\left(-\frac{1}{x}\right)\right], \quad v(x)=x^{2 p-2} w\left(\frac{1}{x}\right) .
$$


To check (11), note that by changing $x$ into $1 / t$,

$$
\begin{aligned}
& \left(\int_{0}^{r} x^{p-2}\left[w\left(\frac{1}{x}\right)+w\left(-\frac{1}{x}\right)\right] d x\right)^{1 / p}\left(\int_{r}^{\infty}\left[x^{2 p-2} w\left(\frac{1}{x}\right)\right]^{-p^{\prime} / p} d x\right)^{1 / p^{\prime}} \\
& \leqslant\left(\int_{|x|<r}|x|^{p-2} w\left(\frac{1}{x}\right) d x\right)^{1 / p}\left(\int_{|x|>r}|x|^{-2} w\left(\frac{1}{x}\right)^{-p^{\prime} / p} d x\right)^{1 / p^{\prime}} \\
& \quad=\left(\int_{|t|>1 / r}|t|^{-p} w(t) d t\right)^{1 / p}\left(\int_{|t|<1 / r} w(t)^{-p^{\prime} / p} d t\right)^{1 / p^{\prime}},
\end{aligned}
$$

which by Lemma A for $\alpha=p$ and the fact that $w \in A_{p}$ with constant $A$ is bounded by

$$
\left(C\left(r^{p} \int_{|t|<1 / r} w(t) d t\right)^{1 / p}\right)\left(\int_{|t|<1 / r} w(t)^{-p^{\prime} / p} d t\right)^{1 / p^{\prime}} \leqslant C A^{1 / p} .
$$

Similarly,

$$
\mathrm{II}+\mathrm{IV}=\int_{0}^{\infty}\left(\int_{x}^{\infty} g(-t) d t\right)^{p} x^{p-2}\left(w\left(\frac{1}{x}\right)+w\left(-\frac{1}{x}\right)\right) d x
$$

is bounded by

$$
\int_{0}^{\infty} g(-x)^{p} x^{2 p-2} w\left(-\frac{1}{x}\right) d x=\int_{-\infty}^{0} g(x)^{p}|x|^{2 p-2} w\left(\frac{1}{x}\right) d x,
$$

since (11) is satisfied for

$$
u(x)=x^{p-2}\left(w\left(\frac{1}{x}\right)+w\left(-\frac{1}{x}\right)\right) \text { and } v(x)=x^{2 p-2} w\left(-\frac{1}{x}\right)
$$

and the lemma follows.

REMARK 1 . The hypothesis in Lemma 1 that $w \in A_{p}$ is unnecessarily strong. As shown in the proof (see (15)), it is enough to assume that

$$
\left(\int_{|t|>s} \frac{w(t)}{|t|^{p}} d t\right)\left(\int_{|t|<s} w(t)^{-1 /(p-1)} d t\right)^{p-1} \leqslant C
$$

for all $s>0$. It follows, for example, that the conclusion of Lemma 1 holds if $w(1 / x)$ is replaced by $w(1 /|x|)$, assuming only that

$$
\left(\int_{s}^{\infty} \frac{w(t)}{t^{p}} d t\right)\left(\int_{0}^{s} w(t)^{-1 /(p-1)} d t\right)^{p-1} \leqslant C
$$

for $s>0$.

More generally, we have

Lemma 1a. If $1<p \leqslant q<\infty$ and $w^{q / p} \in A_{1+q / p^{\prime}}$, then

$$
\begin{gathered}
\left(\int_{-\infty}^{\infty}\left\{|x|^{1 / p^{\prime}-1 / q} w\left(\frac{1}{x}\right)^{1 / p} \int_{|t|>|x|} g(t) d t\right\}^{q} d x\right)^{1 / q} \\
\leqslant C\left(\int_{-\infty}^{\infty} g(x)^{p}|x|^{2 p-2} w\left(\frac{1}{x}\right) d x\right)^{1 / p}
\end{gathered}
$$

for all $g(x) \geqslant 0$. 
Proof. The proof is the same as the previous one, using Hardy's inequality for the pair of weights $u, v$ defined by

$$
u(x)=x^{q / p^{\prime}-1}\left[w\left(\frac{1}{x}\right)^{q / p}+w\left(-\frac{1}{x}\right)^{q / p}\right], \quad v(x)=x^{2 p-2} w\left( \pm \frac{1}{x}\right) .
$$

To verify (11) for this choice of $u$ and $v$, we use the fact that Lemma A holds for $w^{q / p}$ with $\alpha=1+q / p^{\prime}$ since $w^{q / p} \in A_{1+q / p^{\prime}}$. Moreover, as noted in the introduction, (4) holds.

REMARK 1a. As the proof shows, the conclusion of Lemma 1a holds if $w$ merely satisfies

$$
\left(\int_{|t|>s} \frac{w(t)^{q / p}}{|t|^{1+q / p^{\prime}}} d t\right)^{p / q}\left(\int_{|t|<s} w(t)^{-1 /(p-1)} d t\right)^{p-1} \leqslant C
$$

for $s>0$. Thus, the conclusion holds with $w(1 / x)$ replaced by $w(1 /|x|)$, assuming only that

$$
\left(\int_{s}^{\infty} \frac{w(t)^{q / p}}{t^{1+q / p^{\prime}}} d t\right)^{p / q}\left(\int_{0}^{s} w(t)^{-1 /(p-1)} d t\right)^{p-1} \leqslant C .
$$

LemMa 2. If $1<p<\infty$ and $w \in A_{p}$, then

$$
\int_{-\infty}^{\infty}\left(\int_{|t|<|x|} h(t) d t\right)^{p}|x|^{-2} w\left(\frac{1}{x}\right) d x \leqslant C \int_{-\infty}^{\infty} h(x)^{p}|x|^{p-2} w\left(\frac{1}{x}\right) d x
$$

for $h(x) \geqslant 0$.

Proof. The argument is similar to that in the proof of Lemma 1. The integral on the left of (16) is at most $2^{p}$ times

$$
\int_{0}^{\infty}\left(\int_{0}^{x}\right)^{p}+\int_{0}^{\infty}\left(\int_{-x}^{0}\right)^{p}+\int_{-\infty}^{0}\left(\int_{0}^{-x}\right)^{p}+\int_{-\infty}^{0}\left(\int_{x}^{0}\right)^{p}=\mathrm{I}+\mathrm{II}+\mathrm{III}+\mathrm{IV},
$$

and

$$
\begin{aligned}
\mathrm{I}+\mathrm{III} & =\int_{0}^{\infty}\left(\int_{0}^{x} h(t) d t\right)^{p} x^{-2}\left[w\left(\frac{1}{x}\right)+w\left(-\frac{1}{x}\right)\right] d x \\
\mathrm{II}+\mathrm{IV} & =\int_{0}^{\infty}\left(\int_{0}^{x} h(-t) d t\right)^{p} x^{-2}\left[w\left(\frac{1}{x}\right)+w\left(-\frac{1}{x}\right)\right] d x
\end{aligned}
$$

Therefore, in order to prove (16), hypothesis (13) has to be checked for the pair $u, v$ defined by

$$
u(x)=x^{-2}\left[w\left(\frac{1}{x}\right)+w\left(-\frac{1}{x}\right)\right] \text { and } \quad v(x)=x^{p-2} w\left( \pm \frac{1}{x}\right) .
$$

By changing $x$ into $1 / t$.

$$
\begin{aligned}
\left(\int_{r}^{\infty} x^{-2}\left[w\left(\frac{1}{x}\right)+w\left(-\frac{1}{x}\right)\right]\right. & d x)^{1 / p}\left(\int_{0}^{r}\left[x^{p-2} w\left( \pm \frac{1}{x}\right)\right]^{-p^{\prime} / p} d x\right)^{1 / p^{\prime}} \\
& \leqslant\left(\int_{|t|<1 / r} w(t) d t\right)^{1 / p}\left(\int_{|t|>1 / r}|t|^{-p^{\prime}} w(t)^{-p^{\prime} / p} d t\right)^{1 / p^{\prime}} .
\end{aligned}
$$


Since the condition that $w \in A_{p}$ is equivalent to $w^{-p^{\prime} / p} \in A_{p^{\prime}}$, we see by Lemma A applied to $w^{-p^{\prime} / p}$ and $p^{\prime}$, with $\alpha=p^{\prime}$, that the last term is bounded by

$$
C\left(\int_{|t|<1 / r} w(t) d t\right)^{1 / p}\left(r^{p^{\prime}} \int_{|t|<1 / r} w(t)^{-p^{\prime} / p} d t\right)^{1 / p^{\prime}} \leqslant C A^{1 / p},
$$

and the lemma follows.

REMARK 2. The conclusion of Lemma 2 holds if instead of assuming that $w \in A_{p}$, we assume (see (17))

$$
\left(\int_{|t|<s} w(t) d t\right)\left(\int_{|t|>s} \frac{w(t)^{-1 /(p-1)}}{|t|^{p^{\prime}}} d t\right)^{p-1} \leqslant C
$$

for all $s>0$. Hence, the conclusion holds with $w(1 / x)$ replaced by $w(1 /|x|)$ assuming only that

$$
\left(\int_{0}^{s} w(t) d t\right)\left(\int_{s}^{\infty} \frac{w(t)^{-1 /(p-1)}}{t^{p^{\prime}}} d t\right)^{p-1} \leqslant C, \quad s>0 .
$$

LeMma 2a. If $1<p \leqslant q<\infty$ and $w^{q / p} \in A_{1+q / p^{\prime}}$, then

$$
\begin{gathered}
\left(\int_{-\infty}^{\infty}\left\{|x|^{-1 / p-1 / q} w\left(\frac{1}{x}\right)^{1 / p} \int_{|t|<|x|} h(t) d t\right\}^{q} d x\right)^{1 / q} \\
\leqslant C\left(\int_{-\infty}^{\infty} h(x)^{p}|x|^{p-2} w\left(\frac{1}{x}\right) d x\right)^{1 / p}
\end{gathered}
$$

for all $h(x) \geqslant 0$.

Proof. As usual, it is enough to check (13) for

$$
u(x)=x^{-q / p-1}\left[w\left(\frac{1}{x}\right)^{q / p}+w\left(-\frac{1}{x}\right)^{q / p}\right] \text { and } v(x)=x^{p-2} w\left( \pm \frac{1}{x}\right)
$$

In fact, changing $x$ into $1 / t$,

$$
\begin{aligned}
\left(\int_{r}^{\infty} u(x)\right. & d x)^{1 / q}\left(\int_{0}^{r} v(x)^{-p^{\prime} / p} d x\right)^{1 / p^{\prime}} \\
& \leqslant\left(\int_{|t|<1 / r}|t|^{q / p-1} w(t)^{q / p} d t\right)^{1 / q}\left(\int_{|t|>1 / r}|t|^{-p^{\prime}} w(t)^{-p^{\prime} / p} d t\right)^{1 / p^{\prime}} .
\end{aligned}
$$

The first factor on the right is at most

$$
\left(r^{-q / p+1} \int_{|t|<1 / r} w(t)^{q / p} d t\right)^{1 / q}
$$

since $q / p \geqslant 1$, and the second is bounded by

$$
C\left(r^{p^{\prime}} \int_{|t|<1 / r} w(t)^{-p^{\prime} / p} d t\right)^{1 / p^{\prime}}
$$


by Lemma A applied to $w^{-p^{\prime} / p} \in A_{p^{\prime}}$ with $\alpha=p^{\prime}$. (Recall that $w^{q / p} \in A_{1+q / p^{\prime}}$ means that $w \in A_{p} \cap \mathrm{RH}_{q / p}$; in particular, $w \in A_{p}$, and so $w^{-p^{\prime} / p} \in A_{p^{\prime}}$.) Thus, (18) is bounded by

$$
C\left(r \int_{|t|<1 / r} w(t)^{q / p} d t\right)^{1 / q}\left(r \int_{|t|<1 / r} w(t)^{-p^{\prime} / p} d t\right)^{1 / p^{\prime}},
$$

which is at most a constant independent of $r$ since $w^{q / p} \in A_{1+q / p^{\prime}}$.

REMARK 2a. The conclusion of Lemma 2a holds if

$$
\left(\int_{|t|<s}|t|^{q / p-1} w(t)^{q / p} d t\right)^{p / q}\left(\int_{|t|>s} \frac{w(t)^{-1 /(p-1)}}{|t|^{p^{\prime}}} d t\right)^{p-1} \leqslant C, \quad s>0,
$$

as can be seen from (18). In particular, the conclusion holds with $w(1 / x)$ replaced by $w(1 /|x|)$ if

$$
\left(\int_{0}^{s} t^{q / p-1} w(t)^{q / p} d t\right)^{p / q}\left(\int_{s}^{\infty} \frac{w(t)^{-1 /(p-1)}}{t^{p^{\prime}}} d t\right)^{p-1} \leqslant C, \quad s>0 .
$$

3. Proofs of Theorems 1 and 1a. We first prove Theorem 1. For an integrable $f$ with $\int_{-\infty}^{\infty} f=0$, we can write

$$
\hat{f}(x)=\int_{-\infty}^{\infty} f(y)\left(e^{i x y}-1\right) d y=\int_{|x y|<1}+\int_{|x y|>1}=\mathrm{I}+\mathrm{II} .
$$

Using the estimate $\left|e^{i x y}-1\right| \leqslant \operatorname{Min}\{|x y|, 2\}$ and letting $y=1 / t$, we obtain

$$
|\mathrm{I}| \leqslant \int_{|y|<1 /|x|}|x y||f(y)| d y=|x| \int_{|t|>|x|}\left|t^{-3} f(1 / t)\right| d t
$$

and

$$
|\mathrm{II}| \leqslant 2 \int_{|y|>1 /|x|}|f(y)| d y=2 \int_{|t|>|x|}\left|t^{-2} f(1 / t)\right| d t .
$$

If we estimate $|\hat{f}(x)|$ in (2) by the sum of these expressions, apply Lemmas 1 and 2 with $g(t)=\left|t^{-3} f(1 / t)\right|$ and $h(t)=\left|t^{-2} f(1 / t)\right|$, respectively, and then make the change of variables $y=1 / x$, we obtain

$$
\begin{aligned}
\int_{-\infty}^{\infty}|\hat{f}(x)|^{p}|x|^{-2} w\left(\frac{1}{x}\right) d x \\
\leqslant C \int_{-\infty}^{\infty}\left(\int_{|t|>|x|} g(t) d t\right)^{p}|x|^{p-2} w\left(\frac{1}{x}\right) d x \\
\quad+C \int_{-\infty}^{\infty}\left(\int_{|t|<|x|} h(t) d t\right)^{p}|x|^{-2} w\left(\frac{1}{x}\right) d x \\
\leqslant C \int_{-\infty}^{\infty} g(y)^{p}|y|^{2 p-2} w\left(\frac{1}{y}\right) d y+C \int_{-\infty}^{\infty} h(y)^{p}|y|^{p-2} w\left(\frac{1}{y}\right) d y \\
=C \int_{-\infty}^{\infty}|f(x)|^{p}|x|^{p} w(x) d x .
\end{aligned}
$$

This completes the proof. 
To prove Theorem 1a, note that for $f \in \mathscr{S}_{0,0}$ and any fixed positive integer $k$,

$$
\hat{f}(x)=\int_{-\infty}^{\infty} f(y)\left[e^{i x y}-\sum_{j=0}^{k-1} \frac{(i x y)^{j}}{j !}\right] d y=\int_{|x y|<1}+\int_{|x y|>1}=\mathrm{I}+\mathrm{II} .
$$

Again, setting $y=1 / t$, we have

$$
\begin{aligned}
& |\mathrm{I}| \leqslant C \int_{|x y|<1}|x y|^{k}|f(y)| d y=C|x|^{k} \int_{|t|>|x|}\left|t^{-k-2} f(1 / t)\right| d t, \\
& |\mathrm{II}| \leqslant C \int_{|x y|>1}|x y|^{k-1}|f(y)| d y=C|x|^{k-1} \int_{|t|<|x|}\left|t^{-k-1} f(1 / t)\right| d t .
\end{aligned}
$$

The theorem follows from these estimates as before, except that instead of Lemmas 1 and 2 we now use Lemmas $1 \mathrm{a}$ and $2 \mathrm{a}$ with $g(t)=\left|t^{-k-2} f(1 / t)\right|$ and $h(t)=$ $\left|t^{-k-1} f(1 / t)\right|$, respectively.

4. Extensions. Let $W(x)=|x|^{k p} w(x)$, where $k$ is a positive integer and $w$ is a weight such that $w \in A_{p}, 1<p<\infty$. If we define

$$
\mathscr{F} f(x)=\int_{-\infty}^{\infty} f(y)\left[e^{i x y}-\sum_{j=0}^{k-1} \frac{(i x y)^{j}}{j !}\right] d y,
$$

then the proof above shows in particular that the integral defining $\mathscr{F} f$ converges absolutely almost everywhere for any $f \in L^{p}(W)$. We will now show that $\mathscr{F} f(x)$ converges absolutely for all $x$ for every $f \in L^{p}(W)$, with $W$ as above. If $x=0$, the integrand is zero. For any fixed $x \neq 0$, there are constants $C=C_{x}>0$ such that

$$
\begin{aligned}
|\mathscr{F} f(x)| & \leqslant C \int_{|y|<C}\left|y^{k} f(y)\right| d y+C \int_{|y|>C}\left|y^{k-1} f(y)\right| d y \\
& \leqslant C\|f\|_{L^{p}(W)}\left\{\left(\int_{|y|<C} w^{-p^{\prime} / p} d y\right)^{1 / p^{\prime}}+\left(\int_{|y|>C}|y|^{\left.-p^{\prime} w^{-p^{\prime} / p} d y\right)^{1 / p^{\prime}}}\right\}\right.
\end{aligned}
$$

by Hölder's inequality. Thus, $|\mathscr{F} f(x)| \leqslant C\|f\|_{L^{p}(W)}<\infty, C=C_{x, w}$.

Furthermore, since $\mathscr{S}_{0,0}$ is dense in such $L^{p}(W)$ (see [5]), if $\left\{f_{n}\right\} \subset \mathscr{S}_{0,0}$ converges to $f$ in $L^{p}(W)$, then $\mathscr{F} f(x)=\lim _{n \rightarrow \infty} \hat{f}_{n}(x)$ for every $x \in \mathbf{R}$ : in fact, by the previous argument,

$$
\begin{aligned}
\left|\mathscr{F} f(x)-\hat{f}_{n}(x)\right| & =\left|\int_{-\infty}^{\infty}\left[f(y)-f_{n}(y)\right]\left[e^{i x y}-\sum_{j=0}^{k-1} \frac{(i x y)^{j}}{j !}\right] d y\right| \\
& \leqslant C\left\|f-f_{n}\right\|_{L^{p}(W)}, \quad C=C_{x, w},
\end{aligned}
$$

and the right side tends to 0 as $n \rightarrow \infty$. Thus, by Fatou's lemma, we obtain under the hypotheses of Theorem 1a that

$$
\left(\int_{-\infty}^{\infty}\left|\frac{\mathscr{F} f(x)}{x^{k-1}}\right|^{q}\left[\frac{1}{|x|} w\left(\frac{1}{x}\right)\right]^{q / p} \frac{d x}{|x|}\right)^{1 / q} \leqslant C\left(\int_{-\infty}^{\infty}\left|x^{k} f(x)\right|^{p} w(x) d x\right)^{1 / p}
$$

for any $f \in L^{p}\left(|x|^{k} w\right)$, with $C$ independent of $f$. 
For weights of the form $W(x)=|x-a|^{k p} w(x)$ (see the right side of (6)), it is necessary to use a variant of $\mathscr{F} f$ in order to obtain an inequality like (19). In fact, if for given $a$ we define

$$
\mathscr{F}_{a} f(x)=\int_{-\infty}^{\infty} f(y)\left[e^{i x y}-\mathscr{P}_{k-1, a, x}(y)\right] d y,
$$

where

$$
\mathscr{P}_{k-1, a, x}(y)=\sum_{j=0}^{k-1} \frac{(i x)^{j} e^{i x a}}{j !}(y-a)^{j}
$$

is the Taylor polynomial around $a$ of $e^{i x y}$ as a function of $y$, it follows by changing variables that if $w \in A_{p} \cap \mathrm{RH}_{q / p}$, then

$$
\begin{aligned}
& \left(\int_{-\infty}^{\infty}\left|\frac{\mathscr{F}_{a} f(x)}{x^{k-1}}\right|^{q}\left[\frac{1}{|x|} w\left(a+\frac{1}{x}\right)\right]^{q / p} \frac{d x}{|x|}\right)^{1 / q} \\
& \leqslant C\left(\int_{-\infty}^{\infty}|f(x)|^{p}|x-a|^{k p} w(x) d x\right)^{1 / p}
\end{aligned}
$$

with $C$ independent of $a$ and $f$.

Inequality (20) is actually valid assuming only that $w(x+a)$ satisfies (5). This will follow as before if we show that $\mathscr{S}_{0,0}$ is dense in $L^{p}\left(|x-a|^{k p} w\right)$ for such $w$. It suffices to consider the case $a=0$. By Theorem (6.19) of [5], since $w$ is locally integrable, we have only to show that

$$
\frac{1}{n^{p}} \int_{|x|<n} w(x) d x \rightarrow 0 \text { as } n \rightarrow \infty .
$$

We assume that

$$
\int_{|x|>1} \frac{w(x)^{q / p}}{|x|^{1+q / p^{\prime}}} d x<\infty
$$

(see the first factor in the first inequality of (5)). For $N$ large, write

$$
\frac{1}{n^{p}} \int_{|x|<n} w d x=\frac{1}{n^{p}} \int_{|x|<N} w d x+\frac{1}{n^{p}} \int_{N<|x|<n} w d x=A+B .
$$

Clearly, $A \rightarrow 0$ as $n \rightarrow \infty$ for any fixed $N$. By Hölder's inequality,

$$
\begin{aligned}
B & \leqslant \frac{1}{n^{p}}\left(\int_{N<|x|<n} w^{q / p} d x\right)^{p / q}(2 n)^{1-p / q} \\
& \leqslant C\left(\int_{|x|>N} \frac{w(x)^{q / p}}{|x|^{1+q / p^{\prime}}} d x\right)^{p / q} .
\end{aligned}
$$

Thus, $B \rightarrow 0$ uniformly in $n$ as $N \rightarrow \infty$, and the result follows.

5. A counterexample. In this section, we show that Theorem 1 fails for $w(x)=$ $1 /|x|$, i.e., that inequality (1) fails for $\gamma=p-1$ for the class of $f$ with integral zero. In fact, we will show that no norm inequality of the form

$$
\left(\int_{-\infty}^{\infty}|\hat{f}(x)|^{q} u(x) d x\right)^{1 / q} \leqslant C\left(\int_{-\infty}^{\infty}|f(x)|^{p}|x|^{p-1} d x\right)^{1 / p},
$$


$1<p<\infty, 0<q \leqslant \infty, u(x) \not \equiv 0$, can hold for all $f$ with integral zero. It will then follow immediately from the density result in Theorem (6.1) of [5] that no such inequality can hold for all $f \in \mathscr{S}_{0,0}$. A similar statement can be made if the weight $|x|^{p-1}$ on the right above is replaced by $|x|^{k p-1}, k=1,2, \ldots$. For simplicity, we consider only $k=1$.

For $N>1$, define

$$
f(x)=f_{N}(x)=\frac{1}{x}\left\{\chi_{(1 / N, 1)}(x)-\chi_{(1, N)}(x)\right\},
$$

where $\chi_{(a, b)}$ denotes the characteristic function of the interval $(a, b)$. First note that

$$
\int_{-\infty}^{\infty} f d x=\int_{1 / N}^{1} \frac{d x}{x}-\int_{1}^{N} \frac{d x}{x}=\ln N-\ln N=0,
$$

and that

$$
\left(\int_{-\infty}^{\infty}|f(x)|^{p}|x|^{p-1} d x\right)^{1 / p}=\left(\int_{1 / N}^{N} x^{-p} x^{p-1} d x\right)^{1 / p}=(2 \ln N)^{1 / p}
$$

Next, we will show that

$$
|\hat{f}(x)| \geqslant \ln N-2|x|-3 /|x| \text { for all } x .
$$

To see this, write

$$
\begin{aligned}
|\hat{f}(x)| & =\left|\int_{1 / N}^{1} \frac{e^{i x t}}{t} d t-\int_{1}^{N} \frac{e^{i x t}}{t} d t\right|=\left|\int_{1 / N}^{1} \frac{d t}{t}+\int_{1 / N}^{N} \frac{e^{i x t}-1}{t} d t-\int_{1}^{N} \frac{e^{i x t}}{t} d t\right| \\
& \geqslant \int_{1 / N}^{1} \frac{d t}{t}-\left|\int_{1 / N}^{1} \frac{e^{i x t}-1}{t} d t\right|-\left|\int_{1}^{N} \frac{e^{i x t}}{t} d t\right|=\ln N-A-B \text {, say. }
\end{aligned}
$$

Then

$$
A \leqslant \int_{1 / N}^{1} \frac{2|x| t}{t} d t \leqslant 2|x|
$$

and

$$
\begin{aligned}
B & =\left|\int_{1}^{N} \frac{1}{t} \frac{d}{d t}\left\{\frac{e^{i x t}}{i x}\right\} d t\right|=\left|\frac{1}{t} \frac{e^{i x t}}{i x}\right|_{t=1}^{t=N}+\int_{1}^{N} \frac{e^{i x t}}{i x t^{2}} d t \mid \\
& \leqslant \frac{1}{N|x|}+\frac{1}{|x|}+\frac{1}{|x|} \int_{1}^{\infty} \frac{d t}{t} \leqslant \frac{3}{|x|} .
\end{aligned}
$$

Combining estimates leads immediately to (22).

Now, if $u(x) \geqslant 0$ and $u(x) \not \equiv 0$, pick $a, b$ with $0<a<b<\infty$ and $\int_{a<|x|<b} u d x$ $>0$. Then pick $N=N_{a, b}$ so large that, by (23),

$$
|\hat{f}(x)| \geqslant \ln N-2|x|-3 /|x| \geqslant \frac{1}{2} \ln N \text { for } a<|x|<b .
$$

Thus,

$$
\left(\int_{-\infty}^{\infty}|\hat{f}(x)|^{q} u(x) d x\right)^{1 / q} \geqslant\left(\frac{1}{2} \ln N\right)\left(\int_{a<|x|<b} u d x\right)^{1 / q} .
$$

In view of (22) and the fact that $p>1$, this contradicts (21).

We note in passing that a similar construction is given in [4]. 
6. Proof of Theorem 2. Let $f \in \mathscr{S}_{0,0}\left(\mathbf{R}^{n}\right), n>1$. Using $(x \cdot y)$ to denote the ordinary dot product in $\mathbf{R}^{n}$ and noting that $(x \cdot y)^{j}$ is a polynomial in $y$ for $j=0,1, \ldots$, we can write

$$
\begin{aligned}
\hat{f}(x) & =\int_{\mathbf{R}^{n}} f(y)\left[e^{i(x \cdot y)}-\sum_{j=0}^{k-1} \frac{\{i(x \cdot y)\}^{j}}{j !}\right] d y \\
& =\int_{|y|<1 /|x|}+\int_{|y|>1 /|x|}=\mathrm{I}+\mathrm{II} .
\end{aligned}
$$

Since for the change of variables $y=t /|t|^{2}$ the Jacobian is bounded in absolute value by a constant times $|t|^{-2 n}$, we have the estimates

$$
\begin{aligned}
& |\mathrm{I}| \leqslant C|x|^{k} \int_{|y|<1 /|x|}|y|^{k}|f(y)| d y \leqslant C|x|^{k} \int_{|t|>|x|}|t|^{-k-2 n}\left|f\left(\frac{t}{|t|^{2}}\right)\right| d t, \\
& |\mathrm{II}| \leqslant C|x|^{k-1} \int_{|y|>1 /|x|}|y|^{k-1}|f(y)| d y \leqslant C|x|^{k-1} \int_{|t|<|x|}|t|^{-k+1-2 n}\left|f\left(\frac{t}{|t|^{2}}\right)\right| d t .
\end{aligned}
$$

Inequality (7) will then follow from showing that both

$$
\left(\int_{\mathbf{R}^{n}}\left(\int_{|t|>|x|}|t|^{-k-2 n}\left|f\left(\frac{t}{|t|^{2}}\right)\right| d t\right)^{q}\left[|x|^{\beta} w\left(\frac{1}{|x|}\right)\right]^{q / p}|x|^{q-n} d x\right)^{1 / q}
$$

and

$$
\left(\int_{\mathbf{R}^{n}}\left(\int_{|t|<|x|}|t|^{-k+1-2 n}\left|f\left(\frac{t}{|t|^{2}}\right)\right| d t\right)^{q}\left[|x|^{\beta} w\left(\frac{1}{|x|}\right)\right]^{q / p}|x|^{-n} d x\right)^{1 / q}
$$

are bounded by the right side of (7). Note that the right side of (7) is equivalent to

$$
\left(\int_{\mathbf{R}^{n}}\left|f\left(\frac{x}{|x|^{2}}\right)\right|^{p}|x|^{-k p-2 n} w\left(\frac{1}{|x|}\right) d x\right)^{1 / p}
$$

To estimate (24), change to polar coordinates $t=\tau t^{\prime}, \tau=|t|$, and $x=\rho x^{\prime}$, $\rho=|x|$; in this way, (24) becomes

$$
\left(\sigma_{n-1} \int_{0}^{\infty}\left(\int_{\rho}^{\infty} \int_{\left|t^{\prime}\right|=1}\left|f\left(\frac{t^{\prime}}{\tau}\right)\right| \tau^{-k-n-1} d \tau d t^{\prime}\right)^{q} \rho^{q+\beta q / p-1} w\left(\frac{1}{\rho}\right)^{q / p} d \rho\right)^{1 / q},
$$

where $\sigma_{n-1}$ is the surface area of the unit ball in $\mathbf{R}^{n}$. Letting

$$
g(\tau)=\int_{\left|t^{\prime}\right|=1}\left|f\left(\frac{t^{\prime}}{\tau}\right)\right| \tau^{-k-n-1} d t^{\prime}
$$

and

$$
\tilde{w}(\rho)=|\rho|^{-(p-1)(n-1)} w(\rho)
$$


and recalling that $\beta=(n-1) p-n$, we may rewrite (27) as a constant times

$$
\left(\int_{0}^{\infty}\left[\rho^{1 / p^{\prime}-1 / q} \tilde{\mathcal{w}}\left(\frac{1}{\rho}\right)^{1 / p} \int_{\rho}^{\infty} g(\tau) d \tau\right]^{q} d \rho\right)^{1 / q} .
$$

Since $\tilde{w}^{q / p} \in A_{1+q / p^{\prime}}\left(\mathbf{R}^{1}\right)$, Lemma 1a implies that this is at most

$$
C\left(\int_{0}^{\infty} g(\rho)^{p} \rho^{2 p-2} \tilde{w}\left(\frac{1}{\rho}\right) d \rho\right)^{1 / p} .
$$

Since by Hölder's inequality

$$
g(\rho)^{p} \leqslant \sigma_{n-1}^{p / p^{\prime}} \int_{\left|x^{\prime}\right|=1}\left|f\left(\frac{x^{\prime}}{\rho}\right)\right|^{p} \rho^{-p(k+n+1)} d x^{\prime},
$$

it follows that (28) is bounded by

$$
C\left(\int_{0}^{\infty} \int_{\left|x^{\prime}\right|=1}\left|f\left(\frac{x^{\prime}}{\rho}\right)\right|^{p} \rho^{-p(k+n-1)-2} \tilde{w}\left(\frac{1}{\rho}\right) d x^{\prime} d \rho\right)^{1 / p} .
$$

This, however, is easily seen to be a multiple of (26), and the estimation of (24) is complete.

To estimate (25), we argue similarly, rewriting (25) in the form

$$
C\left(\int_{0}^{\infty}\left(\rho^{-1 / p-1 / q} \tilde{w}\left(\frac{1}{\rho}\right)^{1 / p} \int_{0}^{\rho} h(\tau) d \tau\right)^{q} d \rho\right)^{1 / q}
$$

where $\tilde{w}$ is as before and

$$
h(\tau)=\int_{\left|t^{\prime}\right|=1}\left|f\left(\frac{t^{\prime}}{\tau}\right)\right| \tau^{-k-n} d t^{\prime} .
$$

Thus, by Lemma $2 \mathrm{a},(25)$ is bounded by

$$
C\left(\int_{0}^{\infty} h(\rho)^{p} \rho^{p-2} \tilde{w}\left(\frac{1}{\rho}\right) d \rho\right)^{1 / p} .
$$

Using Hölder's inequality to estimate $h(\rho)^{p}$ and arguing as before, we see this is at most a constant times (26), and the proof is complete.

\section{REFERENCES}

1. J. S. Bradley, Hardy inequalities with mixed norms, Canad. Math. Bull. 21 (1978), 405-408.

2. M. Cotlar and C. Sadosky, Inégalités à poids pour les coefficients lacunaires de certaines fonctions analytiques, C. R. Acad. Sci. Paris Ser. I Math. 299 (1984), 591-594.

3. R. Hunt, B. Muckenhoupt, and R. Wheeden, Weighted norm inequalities for the conjugate function and Hilbert transform, Trans. Amer. Math. Soc. 176 (1973), 227-251.

4. B. Muckenhoupt and W.-S. Young, $L^{p}$ multipliers with weight $|x|^{k p-1}$, Trans. Amer. Math. Soc. 275 (1983), 623-639.

5. B. Muckenhoupt, R. Wheeden, and W.-S. Young, $L^{2}$ multipliers with power weights, Adv. in Math. 49 (1983), 170-216.

6. E. M. Stein, Interpolation of linear operators, Trans. Amer. Math. Soc. 83 (1956), 482-492.

Department of Mathematics, Howard University, Washington, D.C. 20059

Department of Mathematics, Rutgers University, New Brunswick, New Jersey 08903 